\title{
POBREZA Y DESERCIÓN ESCOLAR EN EL NIVEL SECUNDARIA EN YUCATÁN
}

\author{
POVERTY AND SCHOOL DROPOUT IN THE SECONDARY LEVEL \\ IN YUCATAN
}

Danahe Casais Molina

Universidad Autónoma de Yucatán, México danahe81@hotmail.com

\section{Isaac Ortega}

Universidad Autónoma de Yucatán, México jisaaco@icloud.com

\section{RESUMEN}

Este artículo explora la relación entre los niveles pobreza y los indicadores educativos de deserción escolar y eficiencia terminal a nivel secundaria mediante un análisis estadístico multivariado en los 106 municipios que conforman el estado de Yucatán, México. Con información del Consejo Nacional de Evaluación de la Política de Desarrollo Social, Coneval, sobre los niveles de pobreza y los indicadores de deserción escolar y eficiencia terminal de la Secretaría de Educación Pública, SEP, se realiza un análisis de componentes principales categórico, al explorar asociaciones entre variables de pobreza y escolaridad de los municipios, así como su relación con cada municipio como caso. Nuestros hallazgos revelan que la pobreza moderada se asocia con la deserción tanto de hombres como de mujeres. Así mismo, los hombres son los que se reincorporan a la escuela en los niveles de pobreza moderada, a diferencia de las mujeres que lo hacen siempre y cuando estén fuera de la pobreza. Se presentan casos particulares de aquellos municipios con los niveles más significativos de pobreza extrema, pobreza moderada, deserción escolar y eficiencia terminal. 
Los hallazgos confirman teorías sobre apoyo condicionado en Yucatán, en donde comunidades en situación de pobreza extrema y beneficiarios del programa Oportunidades consiguen mantener niveles de permanencia altos en la educación secundaria, en tanto que en aquellas en pobreza moderada los estudiantes deben recurrir a otras alternativas, como incorporarse al mercado laboral o permanecer en el hogar para beneficio de la economía familiar, con resultados de deserción escolar.

Palabras clave: pobreza, deserción escolar, eficiencia terminal, nivel secundaria, Yucatán.

Clasificación JEL: I21, I32

\begin{abstract}
In Latin America the abandonment and dropout remain mainly affecting poor and vulnerable students of the various companies in compulsory basic education levels, as in the case of secondary school (Roman, 2009). This study explores the relationship between poverty levels and educational indicators dropout and completion rates at the secondary level by multivariate statistical analysis in the 106 municipalities of the state of Yucatan, Mexico. With Coneval, information poverty levels and indicators of school dropout and completion rates of SEP, we performed a categorical principal components analysis, exploring associations between poverty and schooling variables of municipalities, as well as their relationship to each municipality as appropriate. Our findings reveal that moderate poverty is associated with dropping out of both men and women. Also men are those returning to school again in moderate poverty levels unlike women, who do long as they are out of poverty. We present special cases of those municipalities with significant levels of extreme poverty, moderate poverty, school dropout and completion rates. The findings confirm theories in Yucatan conditional support in communities where extreme poverty and program beneficiaries Oportunidades stay able to maintain high levels of
\end{abstract}


secondary education, while those in moderate poverty, students must turn to other alternatives such as enter the labor market or stay home for the benefit of the family economy dropout results.

Key words: poverty, school dropout, completition rates, secondary school and Yucatán.

\section{INTRODUCCIÓN}

El Consejo Nacional de Evaluación de la Política de Desarrollo Social (Coneval), a partir de la Ley General del Desarrollo Social (LGDS) de 2009, presentó una perspectiva multidimensional para la medición de la pobreza. En este sentido, dicha iniciativa de ley permitió generar información relevante respecto a la situación de pobreza y de bienestar económico de la población en México.

En el año 2012 en nuestro país se registró que 45.5\% de la población se encontraba en situación de pobreza, es decir, 53.3 millones de personas, de las cuales 11.5 millones se ubican en pobreza extrema (Coneval, 2013).

Con el análisis de esta información del Coneval, así como de aquella que proporcionan otros organismos gubernamentales, es posible conocer qué aspectos sociales y políticos están asociados a los distintos niveles de pobreza en cada municipio y estado.

Por ejemplo, un elemento que se menciona como importante para favorecer el crecimiento económico de los países (Orazem y King, 2008) y reducir la pobreza es la educación. Sin embargo, en América Latina el abandono y la deserción escolar están afectando principalmente a los estudiantes más pobres y vulnerables de las distintas sociedades, y es en el nivel secundaria en donde destaca esta situación de vulnerabilidad (Roman, 2009). En dichos grupos, las tasas de repetición y de deserción son significativamente más altas que en las familias de mejores ingresos, diferencias que también siguen siendo significativas entre estudiantes de zonas rurales y urbanas.

Las cifras regionales muestran importantes diferencias al interior de los países, al poner desafíos distintos a las sociedades, los gobiernos y los 
sistemas educativos para atender a los estudiantes en riesgo y disminuir de manera significativa las tasas de deserción.

En México, según datos del Instituto Nacional para la Evaluación de la Educación (INEE, 2009) para el año 2005 en el nivel secundaria se encontraron dificultades en el sector social rural para continuar estudiando. Del total de adolescentes, $14 \%$ no tenían a su alcance los servicios educativos de este nivel básico y, al encontrarse en localidades aisladas o cercanas a la carretera, no podían asistir. En el estado de Yucatán el rezago se ubica precisamente en este grupo de adolescentes, que al tener la edad esperada para cursar la secundaria (12-15 años) no asiste a la escuela.

Distintos organismos, así como investigadores independientes han abordado las variables pobreza y educación al analizar sus indicadores y relacionándolos con aspectos como género, ingreso en los hogares y desarrollo económico (Coneval, 2012a; Pick y Sirkin, 2010; Abril, et al. 2008; Becerril, Ortiz y Albornoz, 2012).

También se han encontrado estudios que muestran una relación directa entre los programas educativos y económicos con mecanismos para reducir la pobreza (Handa, et al. 2009). Sin embargo, aún no ha sido analizada la relación entre los niveles de pobreza y la deserción escolar en el nivel educativo de secundaria en cada uno de los municipios del estado.

El presente estudio abordará la relación entre los indicadores de deserción y eficiencia terminal en el nivel secundaria con los niveles de pobreza en el estado de Yucatán, con base en los datos del Coneval para el 2010 se realizará un análisis por cada municipio.

Para tal efecto se realiza un análisis estadístico multivariado en los 106 municipios del el estado de Yucatán, se utilizan como variables los niveles de pobreza y los indicadores educativos de deserción escolar y eficiencia terminal en el nivel secundaria. Así mismo se explica la relación entre estas variables de acuerdo con las estadísticas y conglomerados que maneja la Secretaría de Educación de Yucatán (2011-2012) y el Coneval (2010).

Otro elemento que se considera en este trabajo es la relación existente de los niveles de pobreza a nivel municipal con los indicadores de deserción escolar y eficiencia terminal en el nivel secundaria, se distingue el comportamiento de estas últimas variables según el sexo del estudiante. 
En los apartados 2, 3 y 4 se tiene como objetivo realizar una revisión teórica y estadística del nivel secundaria y sus indicadores educativos, de los niveles de pobreza y la realidad en Yucatán, así como de los factores que inciden en la deserción escolar, al destacar distintos aspectos que pueden incidir en la permanencia dentro del sistema educativo en las comunidades de Yucatán. En los últimos apartados se describe el método y análisis realizados, los resultados obtenidos y la discusión, así como las conclusiones.

\section{LA SITUACIÓN DE LOS INDICADORES EDUCATIVOS A NI- VEL SECUNDARIA EN YUCATÁN}

La educación formal es uno de los principales factores de desarrollo de los individuos; en este sentido es un mecanismo de transmisión y reproducción de conocimientos, habilidades, actitudes y valores que permiten la integración social, económica y cultural (Coneval, 2012b).

Dentro de esta educación formal, la Secretaría de Educación Pública, SEP (2012) considera que la enseñanza básica constituye el nivel educativo más numeroso, en cuanto a matrícula, del Sistema Educativo Nacional. En este nivel los alumnos adquieren los conocimientos y habilidades intelectuales fundamentales con el propósito de facilitar la sistematización de los procesos de aprendizaje y el desarrollo de valores que alienten su formación integral como personas. Se sientan las bases para que los niños y jóvenes puedan desempeñarse activa y comprometidamente en los distintos ámbitos de la vida.

El INEE desde 2003 desarrolló un sistema de indicadores educativos como instrumento para apoyar la evaluación de la calidad de la educación básica y media superior (SEP, 2012) y conocer el impacto que está teniendo al interior de cada estado.

La aplicación de estos indicadores educativos en México ha permitido realizar revisiones sistemáticas (al inicio y fin de cursos, cada sexenio, etcétera) que han dado como resultado directrices y acciones más precisas que inciden en la consistencia de los objetivos definidos, los medios asignados disponibles y las metas esperadas (SEP, 2005). 
El cuadro 1 muestra los indicadores del Sistema Educativo Nacional para el curso escolar 2011-2012 en el nivel básico, que incluye preescolar, primaria y secundaria.

Cuadro 1. Porcentaje de indicadores educativos para 2011-2012

\begin{tabular}{|l|c|c|c|}
\hline \multicolumn{1}{|c|}{ Indicador educativo } & \multicolumn{3}{|c|}{ Curso escolar 2011-2012 } \\
\hline Educación preescolar & Total & Hombres & Mujeres \\
\hline - Atención de 3 años & & & \\
\hline - Atención de 4 años & 46.5 & 45.4 & 47.7 \\
\hline - Atención de 5 años & 98.0 & 102.8 & 104.9 \\
\hline - Atención de 3,4 y 5 años & 82.8 & 82.0 & 83.8 \\
\hline Educación primaria & & & \\
\hline - Cobertura (6 a 12 años) & 102.8 & 102.9 & 102.8 \\
\hline - Deserción & 0.7 & 0.8 & 0.6 \\
\hline - Reprobación & 3.2 & 3.9 & 2.5 \\
\hline - Eficiencia terminal & 95.1 & 94.8 & 95.4 \\
\hline Educación secundaria & & & \\
\hline - Absorción & 97.0 & 97.6 & 96.5 \\
\hline - Cobertura (13 a 15 años) & 96.8 & 96.7 & 96.9 \\
\hline - Deserción & 5.3 & 6.5 & 4.1 \\
\hline - Eficiencia terminal & 84.2 & 80.9 & 87.6 \\
\hline
\end{tabular}

Fuente: En el cálculo de cobertura utilizaron las proyecciones de Conapo, febrero 2007. Para los indicadores de eficiencia terminal, deserción y reprobación las cifras son estimadas de SEP para el ciclo 2011-2012.

El cuadro permite observar que la deserción escolar es mayor en el nivel secundaria, en comparación con los otros niveles de educación básica; también muestra una mayor proporción de la población masculina. Por otro lado, en la eficiencia terminal se reduce el porcentaje de alumnos, las niñas son las que obtienen mejores resultados.

La tríada de indicadores de eficiencia educativa que se plantea en este cuadro, y que resultan las más representativas en relación con el éxito o el fracaso escolar son: reprobación, deserción y eficiencia terminal (SEP, 2005). 
El indicador educativo de eficiencia terminal permite conocer el número de alumnos que finaliza un nivel educativo de manera regular (dentro del tiempo establecido) y el porcentaje de alumnos que lo culminan extemporáneamente. Es la relación porcentual la que resulta de dividir el número de egresados de un nivel educativo determinado entre el número de estudiantes de nuevo ingreso que entraron al primer grado de ese nivel educativo en años previos. Se considera a los egresados como aquellos alumnos que se hacen acreedores a un certificado de terminación de estudios una vez concluido un nivel educativo.

Para este estudio los indicadores que se consideran para la eficiencia terminal son: matrícula y nuevo ingreso. También se consideran los indicadores de reingreso de los alumnos, los aprobados y la existencia de alumnos en el curso escolar 2011-2012 como elementos que inciden en la eficiencia terminal.

Los alumnos de nuevo ingreso son aquellos que ingresan a primer grado en un determinado nivel educativo (SEP, 2006), mientras que el reingreso se refiere a los que se reincorporan a la escuela en cualquier grado del nivel educativo. Los aprobados se refiere al total de alumnos que han acreditado satisfactoriamente las evaluaciones establecidas en los planes y programas de estudio, es decir, el porcentaje de alumnos promovidos al siguiente grado al final del ciclo escolar (SEP, 2005).

La deserción es el indicador educativo que expresa el número o porcentaje de alumnos que abandonan las actividades escolares antes de terminar algún grado o nivel educativo. La deserción se clasifica en tres vertientes: deserción intracurricular, deserción intercurricular y deserción total. El abandono que ocurre durante el ciclo escolar se denomina deserción intracurricular, al que se realiza al finalizar el ciclo escolar, independientemente de que el alumno haya aprobado o no, se le llama deserción intercurricular y la deserción total es la combinación de ambas.

La educación secundaria es el tercero y último nivel que conforma la educación básica. Se cursa en tres grados y es de carácter propedéutico, es decir, es necesaria para ingresar al nivel medio superior y su carácter es obligatorio (SEP, 2012). 
Este nivel es reconocido tanto por su valor intrínseco como por su valor agregado; aporta conocimientos, habilidades y actitudes para que los adolescentes continúen en el sistema educativo y los vaya formando para ingresar en el mercado laboral.

Por lo anterior, existen políticas por parte del estado para poder mantener las matrículas en la secundaria pública, aun en contra de las condiciones socioeconómicas más adversas. Duro, Volpi y Contreras (2010) afirman con certeza que es la oferta de la educación secundaria la que determina el nivel educativo que alcanzará la mayor parte de la población $\mathrm{y}$, por tanto, que define el perfil cultural, económico y ciudadano del país, con las consecuencias individuales y sociales que ello implica.

La SEP reporta que este nivel educativo atiende cinco opciones de servicios: secundaria general, telesecundaria, secundaria técnica, en donde se capacita a los alumnos en alguna actividad tecnológica industrial, comercial, agropecuaria, pesquera o forestal, secundaria para trabajadores y secundaria comunitaria.

En Yucatán, según la matrícula de los ciclos escolares 2010-2011 y 2011-2012 proporcionada por el departamento de estadística de la Secretaría de Educación para este estudio, se han dado cambios poco significativos en cuanto al número de alumnos que asisten a la secundaria.

El cuadro 2 muestra como disminuye la asistencia de alumnos en el nivel secundaria. En primero y segundo grado aumenta la matrícula para el curso 2011-2012; pero para el tercero se reduce significativamente el número de alumnos en relación con los grados anteriores.

También se reporta mayor presencia masculina en la escuela secundaria en relación con la femenina en los tres grados académicos. 
Cuadro 2. Matrícula de alumnos de secundaria en Yucatán, ciclos 2010-2011 y 2011-2012

\begin{tabular}{|c|c|c|}
\hline & \multicolumn{2}{|c|}{ Ciclo escolar } \\
\hline Primero & $\mathbf{2 0 1 0 - 2 0 1 1}$ & $\mathbf{2 0 1 1 - 2 0 1 2}$ \\
Hombres & 34,618 & 35,444 \\
Mujeres & 17,896 & 18,428 \\
\hline Segundo & 16,722 & 17,016 \\
Hombres & 31,928 & 32,882 \\
Mujeres & 16,595 & 16,726 \\
\hline Tercero & 15,333 & 16,156 \\
Hombres & 31,335 & 29,319 \\
Mujeres & 15,820 & 14,884 \\
Total & 15,515 & 14,435 \\
Hombres & 97,881 & 97,645 \\
Mujeres & 50,311 & 50,038 \\
\hline
\end{tabular}

Fuente: Se elaboró de acuerdo con datos del departamento de estadística de la SEP.

Es importante mencionar en la secundaria los adolescentes están ya en edad de ingresar al ámbito laboral. Medio por el cual no terminan su educación, reduciéndose la matrícula del segundo al tercer grado de secundaria en la mayoría de los municipios, en el curso escolar 2011-2012.

\section{LOS NIVELES DE POBREZA EN YUCATÁN}

De acuerdo con el Coneval (2012c) una persona se encuentra en situación de pobreza cuando tiene, al menos, una carencia social (sea rezago educativo, acceso a servicios de salud, acceso a la seguridad social, calidad y espacios de la vivienda, servicios básicos en la vivienda o acceso a la alimentación) y su ingreso es insuficiente para adquirir los bienes y servicios que requiere para satisfacer sus necesidades alimentarias y no alimentarias.

De tal manera que una persona en pobreza extrema es aquella que tiene tres o más carencias de seis posibles, dentro del índice de privación social y que, además, se encuentra por debajo de la línea de bienestar mí- 
nimo. Las personas en dicha situación disponen de un ingreso tan bajo que, aun si lo dedicaran por completo a la adquisición de alimentos, no podrían obtener los nutrientes necesarios para tener una vida sana.

En 2004 se aprueba una Ley General de Desarrollo Social que, al mismo tiempo, crea en 2006 un organismo independiente, Coneval, para medir la pobreza a nivel nacional, estatal y municipal (Coneval, 2012b). Este organismo elabora una nueva metodología que, a diferencia de la anterior, comprende una visión multidimensional de la pobreza.

El cuadro 3 reporta los indicadores de pobreza, privación social y carencia social, que son evaluados por el Coneval en el periodo 2010-2012. En relación con la pobreza se puede ver que la mayor proporción se encuentra en pobreza moderada y que, en relación con 2010, ésta aumentó para el año 2012, a diferencia de la extrema, la cual disminuyó en cuanto al número de personas y carencias promedio.

Cuadro 3. Medición de la pobreza Estados Unidos Mexicanos, 2012 Porcentaje, número de personas y carencias promedio por indicador de pobreza 2010-2012

\begin{tabular}{|l|c|c|c|c|c|c|}
\hline \multicolumn{1}{|c|}{ Indicadores } & \multicolumn{2}{|c|}{$\begin{array}{c}\text { Millones } \\
\text { Porcentaje }\end{array}$} & \multicolumn{2}{c|}{$\begin{array}{c}\text { Carencias } \\
\text { de personas }\end{array}$} \\
promedio \\
\hline Pobreza & $\mathbf{2 0 1 0}$ & $\mathbf{2 0 1 2}$ & $\mathbf{2 0 1 0}$ & $\mathbf{2 0 1 2}$ & $\mathbf{2 0 1 0}$ & $\mathbf{2 0 1 2}$ \\
\hline Población en situación de pobreza & & & & & & \\
\hline Población en situación de pobreza moderada & $\mathbf{4 6 . 1}$ & $\mathbf{4 5 . 5}$ & $\mathbf{5 2 . 8}$ & $\mathbf{5 3 . 3}$ & 2.6 & 2.4 \\
\hline Población en situación de pobreza extrema & 34.8 & 35.7 & 39.8 & 41.8 & 2.2 & 2.0 \\
\hline Población vulnerable por carencias sociales & 11.3 & 9.8 & 13.0 & 11.5 & 3.8 & 3.7 \\
\hline Población vulnerable por ingresos & $\mathbf{2 8 . 1}$ & $\mathbf{2 8 . 6}$ & $\mathbf{3 2 . 1}$ & $\mathbf{3 3 . 5}$ & 1.9 & 1.8 \\
\hline Población no pobre no vulnerable & $\mathbf{5 . 9}$ & $\mathbf{6 . 2}$ & $\mathbf{6 . 7}$ & $\mathbf{7 . 2}$ & 0.0 & 0.0 \\
\hline Indicadores de carencia social & $\mathbf{1 9 . 9}$ & $\mathbf{1 9 . 8}$ & $\mathbf{2 2 . 8}$ & $\mathbf{2 3 . 2}$ & 0.0 & 0.0 \\
\hline Rezago educativo & & & & & & \\
\hline Carencias en el acceso a los servicios de salud & 20.7 & 19.2 & 23.7 & 22.6 & 3.1 & 2.9 \\
\hline Carencia en el acceso a la seguridad social & 29.2 & 21.5 & 33.5 & 25.3 & 3.0 & 2.8 \\
\hline Carencia por calidad y espacios de la vivienda & 60.7 & 61.2 & 69.6 & 71.8 & 2.5 & 2.3 \\
\hline Carencia en el acceso a los servicios básicos de la vivienda & 22.9 & 21.2 & 26.3 & 24.9 & 3.3 & 3.2 \\
\hline Carencia en el acceso a la alimentación & 24.8 & 23.3 & 28.4 & 27.4 & 3.0 & 2.9 \\
\hline
\end{tabular}

Fuente: Estimaciones del Coneval en base en el MCS-ENIGH 2010-2012 (Coneval, 2013). 
Esto se ha debido a que, en general, la política social ha sido dirigida a la población con más pobreza y a que la crisis económica y el incremento en el precio de los alimentos golpearon a la población, especialmente en las áreas urbanas de país (Coneval, 2012b).

Entre 2010 y 2012 se redujo de 13.0 a 11.5 millones el número de personas ubicadas en pobreza extrema, es decir, pasó de 11.3 a 9.8 por ciento. Para 2012 la distribución de la pobreza por entidad federativa ubica al estado de Yucatán en el décimo tercer lugar, en relación con la pobreza en el resto de las entidades federativas y el duodécimo en pobreza extrema. En este último se encuentra en la media nacional (9.8\%).

Ahora bien, respecto a las diferencias en la incidencia de la pobreza por grupo social el Coneval (2012b) refiere que el porcentaje de la población menor a 18 años en situación de pobreza fue de 53.8 en 2010, lo que equivaldría a 21.4 millones de niños y jóvenes, es el grupo de edad con mayor porcentaje de pobreza en el país.

También manifiestan que el porcentaje de quienes residen en las zonas de atención prioritaria que se encuentran en condición de pobreza ascendió a $77.8 \%$ en 2010 , lo que quiere decir que había 13.6 millones de personas en esa condición.

La proporción de hablantes de lengua indígena en condición de pobreza ascendía a 79.3\% en 2010 (la más alta de los grupos considerados en este apartado). Si bien se han presentado avances importantes en coberturas básicas, tanto para quienes viven en zonas de atención prioritaria como para la población indígena, la doble crisis global iniciada en 2007-2008 (de los precios de los alimentos y financiera) parece haber impactado el ingreso de la población en su conjunto. Esto contribuyó al aumento de la pobreza para el año 2010 en población vulnerable, principalmente como los hablantes de lengua indígena. 
Cuadro 4. Porcentaje, número de personas y carencias promedio por indicador de pobreza en Yucatán 2010 y 2012

\begin{tabular}{|l|c|c|c|c|c|c|}
\hline \multicolumn{1}{|c|}{ Indicadores } & \multicolumn{2}{c|}{ Porcentaje } & \multicolumn{2}{c|}{$\begin{array}{c}\text { Miles de } \\
\text { personas }\end{array}$} & $\begin{array}{c}\text { Carencias } \\
\text { promedio }\end{array}$ \\
\hline Pobreza & $\mathbf{2 0 1 0}$ & $\mathbf{2 0 1 2}$ & $\mathbf{2 0 1 0}$ & $\mathbf{2 0 1 2}$ & $\mathbf{2 0 1 0}$ & $\mathbf{2 0 1 2}$ \\
\hline Población en situación de pobreza & & & & & & \\
\hline \multicolumn{1}{|c|}{ Población en situación de pobreza moderada } & 36.6 & 39.0 & 726.1 & 796.3 & 2.3 & 2.4 \\
\hline \multicolumn{1}{|c|}{ Población en situación de pobreza extrema } & 11.7 & 9.8 & 232.5 & 200.6 & 3.8 & 3.7 \\
\hline Población vulnerable por carencias sociales & $\mathbf{2 6 . 0}$ & $\mathbf{2 7 . 0}$ & $\mathbf{5 1 9 . 9}$ & $\mathbf{5 5 1 . 4}$ & 2.0 & 2.1 \\
\hline Población vulnerable por ingresos & $\mathbf{6 . 4}$ & $\mathbf{6 . 3}$ & $\mathbf{1 2 7 . 7}$ & $\mathbf{1 2 7 . 6}$ & - & - \\
\hline Población no pobre no vulnerable & $\mathbf{1 9 . 2}$ & $\mathbf{1 7 . 9}$ & $\mathbf{3 8 1 . 5}$ & $\mathbf{3 6 4 . 6}$ & - & - \\
\hline Indicadores de carencia social & & & & & & \\
\hline Rezago educativo & 24.7 & 23.4 & - & - & - & - \\
\hline Carencias en el acceso a los servicios de salud & 20.7 & 15.7 & - & - & - & - \\
\hline Carencia en el acceso a la seguridad social & 56.9 & 58.8 & - & - & - & - \\
\hline Carencia por calidad y espacios de la vivienda & 19.5 & 20.6 & - & - & - & - \\
\hline Carencia en el acceso a los servicios básicos de la & 37.4 & 42.7 & - & & & \\
vivienda & 31.4 & & - & - & - & - \\
\hline Carencia en el acceso a la alimentación & 21.4 & 25.1 & - & - & - & - \\
\hline
\end{tabular}

Fuente: Estimaciones del Coneval (2013).

El cuadro 4 reporta para Yucatán que el porcentaje de pobreza aumentó ligeramente de $48.3 \%$ a $48.9 \%$, así como la situación de pobreza extrema disminuyó a 9.8 \%, mientras que la pobreza moderada se elevó a 39.0 por ciento. Estos datos nos proporcionan un panorama estadístico de cómo se encuentra el estado de Yucatán en relación con los indicadores de pobreza y cómo se han ido modificando a través de los años. La pobreza en este sentido, por la carencia de acceso a distintos aspectos sociales y económicos, es uno de los factores que tiene una fuerte influencia en la presencia de indicadores de deserción escolar en los sectores menos favorecidos. 


\section{FACTORES QUE INCIDEN EN LA DESERCIÓN ESCOLAR}

La deserción escolar puede estar asociada con distintos aspectos entre los cuales Abril, et al. (2008) destacan los siguientes: económicos, que implican la falta de recursos para sostener los gastos de la escuela, inexistencia o ausencia de oferta educativa para los niveles de secundaria, medio superior y superior, problemas familiares que puedan presentarse en esta etapa de desarrollo, así como las tareas y demandas del hogar, falta de interés y problemas en el desempeño escolar.

En la literatura sobre el tema se reporta la relación entre el nivel educativo con variables como capital social y crecimiento económico, observándose una influencia directa entre ellas (Self y Grabowski, 2004; Petrakis y Stamatakis, 2002). Para Ferguson (2006) el capital social incluye los factores de protección de la familia y de la comunidad, los cuales para el autor tienen una relación directa con el rendimiento académico de los niños. Entre los aspectos de protección se encuentran, apoyo en las tareas del hijo, aspiraciones académicas que se tengan en la familia, apoyo de miembros de la familia extendida $y$, uno de los aspectos principales, la presencia de la madre y sus redes de apoyo social.

En Yucatán se ha encontrado que las zonas rurales son las que presentan mayores dificultades, debido al nivel de ingreso en las familias. Este es un factor que incide en la dinámica que se presenta al interior de los hogares y repercute en los hijos y sus actividades. Alvarado, Leyva y Bolio (2007) reportan, en relación con el ingreso, que las dificultades en cuanto a lo que perciben las familias incide en las actividades que se realicen en la región. De tal manera que surgen distintas actividades tales como: migración en busca de espacios laborales, elaboración de artesanías, urdido de hamacas, pequeños productores de cultivos comerciales, trabajos con bajos salarios, entre otros. Para lo cual el permanecer o no en el sistema educativo no es una prioridad, sino más bien la búsqueda de recursos para satisfacer las necesidades básicas de la familia.

Existen zonas dentro del estado que tienen una importante relación con el sector turístico, lo cual es una fuente importante de ingresos para algunas comunidades y familias. Barkin (2001) considera la actividad tu- 
rística, efectivamente, como una industria que promueve la generación de empleos y divisas; sin embargo, también implica que su crecimiento descontrolado puede provocar graves problemas sociales y ambientales (García de Fuentes y Xool, 2012).

En este sentido, la migración y el mercado laboral son aspectos que propician el abandono de la escuela en edades tempranas. De acuerdo con la SEP (2003) en Yucatán existen comunidades en las cuales niños y adolescentes trabajan para contribuir económicamente y cubrir las necesidades de su familia, lo cual puede estar relacionado con el índice de deserción escolar en el estado.

Se reporta que aquellos niños que por necesidades económicas de las familias trabajan en labores domésticas o extradomésticas, asisten a la escuela con menor frecuencia que sus pares que no trabajan (INEE, 2009). Por lo cual, la asistencia a clases de estos niños o adolescentes se va reduciendo a medida que aumentan las horas en las cuales trabajan (Levinson, Moe, Knaul, 2008). Estos resultados atentan contra las posibilidades de que, los niños que trabajan extensamente, puedan completar su escolaridad básica en el tiempo normativo.

Asimismo, autores como Levinson, Moe, Knaul (2008) y Edmonds (2008) han encontrado que, bajo condiciones de pobreza, los niños dedican menos tiempo a la escuela que al trabajo, con diferencias en relación con el sexo. Es decir, los varones se relacionan con trabajos extemporáneos donde obtienen dinero rápido, mientras que las niñas están en el hogar coordinándose entre la escuela y dichas actividades.

En este sentido Lagarde (2012), al estudiar las cuestiones de diferenciación sexual, menciona que la mujer, a diferencia del hombre, tiene que asumir roles o papeles en la vida doméstica y coordinarlos con los demás aspectos de su vida. De tal manera que las niñas pueden tener mayores probabilidades de dejar la escuela para dedicarse al ámbito familiar.

Otro factor importante que se ha considerado como elemento para reducir la deserción escolar son los programas de becas condicionados al desempeño escolar. En particular, Orazem y King (2008) han señalado que, para reducir la pobreza en aquellas comunidades donde existe una gran demanda de menores como mano de obra, el uso de tales programas podría ser particularmente exitoso. 
En México una opción que ha surgido para apoyar a estos alumnos a que terminen sus estudios es el programa Oportunidades, el cual apoya principalmente a la población en pobreza extrema dándole recursos económicos por hijo. El objetivo del programa es favorecer el desarrollo de capacidades asociadas a salud, nutrición y educación para contribuir a romper el ciclo intergeneracional de la pobreza. Para identificar familias beneficiarias Oportunidades utiliza un sistema de selección basado en características socioeconómicas y demográficas de los hogares e incorpora a los que se encuentran en situaciones de pobreza más acentuadas (Sedesol, 2012).

\section{METODOLOGÍA}

Para la exploración de los datos se consideró el análisis categórico de componentes principales (Categorical Principal Component Analysis, CATPCA), el cual se utiliza para hacer análisis de datos multivariados complejos, en el que se incluyen tanto variables nominales, ordinales o numéricas (Meulman, Van Der Kooij, Heisser, 2004).

En el presente estudio se considera dicho análisis con la intención de representar las relaciones entre las variables y entre estas con cada uno de los municipios del estado de Yucatán, mediante su ubicación en un diagrama de dispersión biespacial (Linting, et al. 2007) en donde las magnitudes y aproximaciones de los vectores determinan los resultados de tales variables. Los análisis fueron realizados con el IBM SPSS Statistics 21.0.

La información utilizada sobre educación corresponde al ciclo escolar 2011-2012, por cada municipio, en cuanto a los indicadores educativos del nivel secundaria: deserción escolar y eficiencia terminal (matrícula y nuevo ingreso), así como los indicadores de reingreso aprobación y existencia, fueron proporcionados por el departamento de estadística del organismo. Se contó con información de los alumnos de las cinco modalidades del nivel en cuestión, es decir: secundaria general, telesecundaria, secundaria técnica, secundaria para trabajadores y secundaria comunitaria.

También se utilizaron los datos estadísticos que son publicados por el Coneval para el año 2010 de acuerdo con los niveles de pobreza por cada 
municipio, ya que para el año 2012 sólo se han publicado los resultados para el nivel estatal y no para el municipal. Dado que la información obtenida del primer organismo se reporta en total de estudiantes por municipio, se dividió dicha cantidad entre la población matriculada en el nivel escolar (secundaria) para cada uno de ellos, para así manejar porcentajes y coincida con el tipo de reportes generados por el segundo organismo.

En particular, las variables utilizadas, definidas conceptualmente en secciones anteriores, para los niveles de pobreza son pobreza extrema y pobreza moderada; mientras que para los indicadores educativos están los índices de aprobación, deserción y sus tres vertientes: deserción intracurricular, deserción intercurricular y deserción total y, por último, las variables asociadas a la eficiencia terminal, que son: matrícula, nuevo ingreso, reingreso, aprobación y existencia.

Como se mencionó anteriormente, en todo caso, la variable fue operacionalizada como la proporción de estudiantes, del total en edad escolar matriculados en el nivel secundaria, para evitar efectos de esta última variable. Indicadores educativos se separaron en dos componentes por sexo y se considera que la realidad de las mujeres es diferente a la de los hombres en cuanto a motivos de deserción y a su papel socioeconómico las comunidades.

\section{RESULTADOS}

Los hallazgos muestran que el indicador total de pobreza, es decir, la suma entre pobreza extrema y moderada, para los municipios con menores índices son: Mocochá (26.8), Mérida (29.4), Río Lagartos (36.7), San Felipe (37.8) y Telchac puerto (37.9). Así como los de mayor índice de población en pobreza total son: Tahdziú (91.7), Chikindzonot (90.0), Mayapán (89.8) Chacsinkín (89.4) y Chemax (89.1).

Para la pobreza extrema los municipios con mayor porcentaje de población son: Tahdziú (56.5), Chikindzonot (51.8), Chemax (50.4), Tixcacalcupul (45.5) y Cantamayec (45.2).

Para los indicadores educativos, de acuerdo con las estadísticas de SEP (2011-2012), se encontró que los índices más altos de deserción total 
(de 24.7 a 16.4) se ubicaron en los municipios de: Dzan, Santa Elena, Chumayel, Dzitás y Dzilam de Bravo, Homún, Tixpeual, Sacalum, Celestún y Conkal. Con menores índices de deserción (de -5.9 a 0) fueron los municipios de: Quintana Roo, Chicxulub, Tepakán, Cuncunul, Chapab, Bokobá, Dzilam González, Ixil, Kaua y Muxupip.

Los indicadores de eficiencia terminal los municipios con menores índices, es decir, aquellos que mostraron porcentajes más bajos (de 26.1 a 63), fueron: Conkal, Celestún, Halachó, Tetiz, Dzitás, Kinchil, Ucu, Dzan, Telchac Pueblo y Dzoncauich. Tanto que los índices más altos de eficiencia terminal (del 100 al 94.7) son los municipios de: Cuncunul, Tekit, Sanahcat, Motul, Temozón, Telchac Pueblo, Mérida, Dzilam González, Yaxkukul y Kaua.

De los resultados anteriores destaca la diferencia que existe entre cada municipio del estado de Yucatán en cuanto a los niveles de pobreza, así como en los indicadores educativos de deserción y los indicadores asociados a la eficiencia terminal.

A partir de estas variables se realiza el análisis exploratorio descriptivo. En el diagrama de dispersión (figura 1) la longitud de las líneas son proporcionales a la magnitud de las variables, mientras más cercanas se encuentren las líneas y sus puntos finales unos de otros mayor similitud existirá entre el comportamiento de estas variables. Por otro lado, la dirección de cada línea no indica, necesariamente, un valor positivo o negativo, solamente representa su posición relativa con respecto a las otras variables, por lo que las dimensiones 1 y 2 sólo son convencionales para fines de la representación. Cada línea punteada representa una variable, sin existir diferencia entre el tipo de línea usada.

De tal manera que, en la gráfica 1 , se observa que la pobreza moderada (POB MOD) se asocia con la deserción intracurricular (Des. Intra) e intercurricular (Des. Inter) tanto de hombres $(\mathrm{H})$ como de mujeres $(\mathrm{M})$, y es más evidente la intracurricular, es decir, la que se realiza durante el ciclo escolar que se está cursando, ya que tal línea tiene una mayor cercanía. Sobresale que los hombres son quienes se reincorporan a la escuela (Reingreso $\mathrm{H}$ ) nuevamente en los niveles de pobreza moderada, a diferencia de las mujeres.

La pobreza extrema (POB EXT), por su parte, se encuentra asociada con las variables de eficiencia terminal, esto es, el nuevo ingreso (N. Ingreso) 
y la matrícula (Matrícula $1^{\circ}, 2^{\circ}$ y $3^{\circ}$ ) en la educación secundaria, tanto en hombres como en mujeres. Así mismo se encuentran los indicadores de Existencia y Aprobados en esta sección del diagrama.

Las mujeres con reingreso a la escuela son, particularmente, las que están fuera de los perfiles de pobreza (Reingreso M), ya que la línea que las representa está en sentido opuesto al sector de pobreza extrema (POB EXT).

Gráfica 1. Mapa de saturación por componentes de variables de niveles de pobreza y de indicadores educativos en los municipios de Yucatán

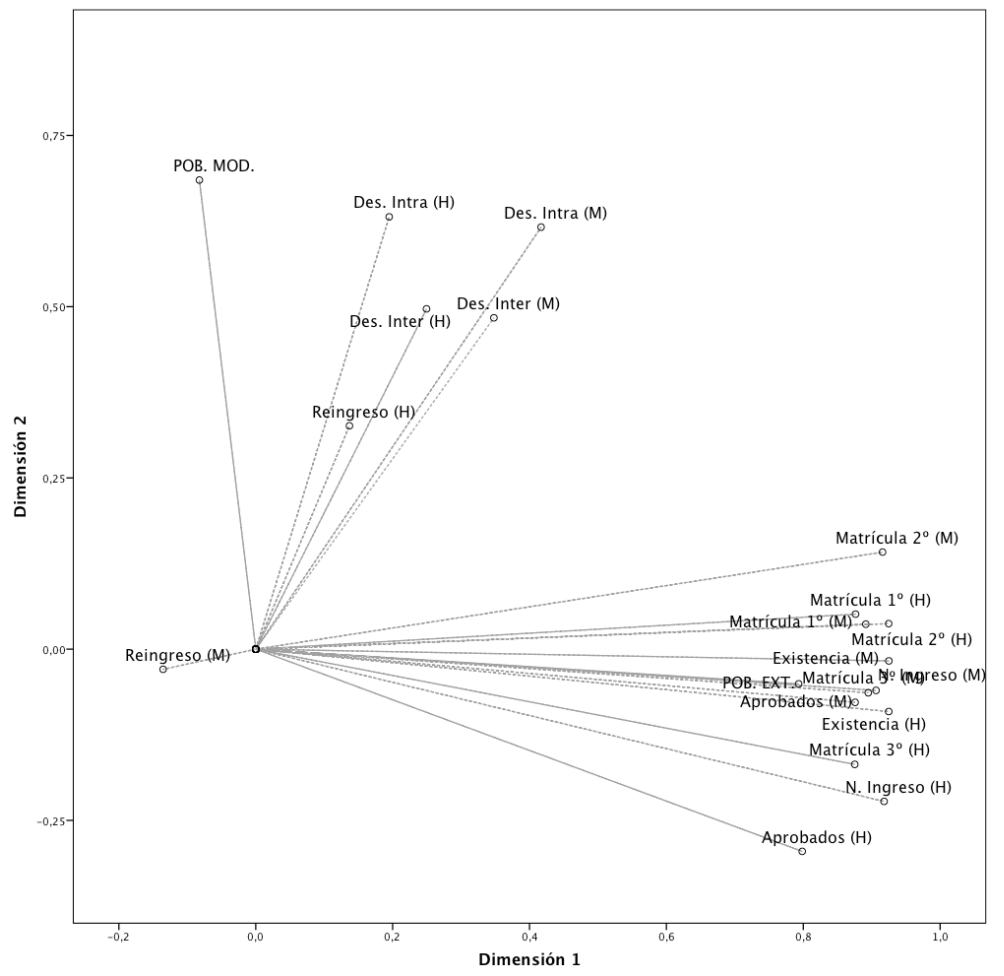

Fuente: Elaborado de acuerdo con los análisis sobre los niveles de pobreza e indicadores educativos de la SEP 2011-2012.

El análisis bidimensional de distancias entre municipios, se realiza en función del número que ocupa cada uno de los municipios del estado de Yucatán, en orden alfabético (cuadro 5). 
Cuadro 5. Relación de municipios del estado de Yucatán con los numerales usados para su identificación en el CATPCA

\begin{tabular}{|c|c|c|c|}
\hline \multicolumn{4}{|c|}{ Municipios } \\
\hline 1. Abalá & 28. Dzilam de Bravo & 55. Opichén & 82. Telchac Pueblo \\
\hline 2. Acanceh & 29. Dzilam González & 56. Oxkutzcab & 83. Telchac Puerto \\
\hline 3. Akil & 30. Dzitás & 57. Panabá & 84. Temax \\
\hline 3. Akil & 30. Dzitás & 57. Panabá & 84. Temax \\
\hline 4. Baca & 31. Dzoncauich & 58. Peto & 85. Temozón \\
\hline 5. Bokobá & 32. Espita & 59. Progreso & 86. Tepakán \\
\hline 6. Buctzotz & 33. Halachó & 60. Quintana Roo & 87. Tetiz \\
\hline 7. Cacalchén & 34. Hocabá & 61. Río Lagartos & 88. Teya \\
\hline 8. Calotmul & 35. Hoctún & 62. Sacalum & 89. Ticul \\
\hline 9. Cansahcab & 36. Homún & 63. Samahil & 90. Timucuy \\
\hline 10. Cantamayec & 37. Huhí & 64. Sanahcat & 91. Tinum \\
\hline 11. Celestún & 38. Hunucmá & 65. San Felipe & 92. Tixcacalcupul \\
\hline 12. Cenotillo & 39. Ixil & 66. Santa Elena & 93. Tixkokob \\
\hline 13. Conkal & 40. Izamal & 67. Seyé & 94. Tixmehuac \\
\hline 14. Cuncunul & 41. Kanasín & 68. Sinanché & 95. Tixpéhual \\
\hline 15. Cuzamá & 42. Kantunil & 69. Sotuta & 96. Tizimín \\
\hline 16. Chacsinkín & 43. Kaua & 70. Sucilá & 97. Tunkás \\
\hline 17. Chankom & 44. Kinchil & 71. Sudzal & 98. Tzucacab \\
\hline 18. Chapab & 45. Kopomá & 72. Suma & 99. Uayma \\
\hline 19. Chemax & 46. Mama & 73. Tahdziú & 100. Ucú \\
\hline 20. Chicxulub Pueblo & 47. Maní & 74. Tahmek & 101. Umán \\
\hline 21. Chichimilá & 48. Maxcanú & 75. Teabo & 102. Valladolid \\
\hline 22. Chikindzonot & 49. Mayapán & 76. Tecoh & 103. Xocchel \\
\hline 23. Chocholá & 50. Mérida & 77. Tekal de Venegas & 104. Yaxcabá \\
\hline 24. Chumayel & 51. Mocochá & 78. Tekantó & 105. Yaxkukul \\
\hline 25. Dzán & 52. Motul & 79. Tekax & 106. Yobaín \\
\hline 26. Dzemul & 53. Muna & 80. Tekit & \\
\hline 27. Dzidzantún & 54. Muxupip & 81. Tekom & \\
\hline
\end{tabular}

Fuente: INEGI (2014). 
En la gráfica 2 se presenta el mapeo de los indicadores de pobreza (POB MOD y POB EXT), los de deserción (Des. Intra y Des. Inter), los de eficiencia terminal (Matrícula y N. Ingreso), así como los indicadores de Existencia y Aprobados, en relación con los 106 municipios del estado, de tal manera que cada uno de ellos se ubica en determinado sector del plano.

Se observa que los municipios que se encuentran más cercanos tanto a la pobreza moderada como a los indicadores de deserción intracurricular e intercurricular son 66: Santa elena, 25: Dzan, 11: Celestún, 21: Chichimilá, 77: Tekal de Venegas, 32: Espita, 96: Tizimín, 30: Dzitás y 68: Sinanché.

Para el caso de la dimensión de pobreza extrema, existe la relación directa con matrícula e ingreso en los municipios, 71: Sudzal, 16: Chacsinkin, 10: Cantamayec, 19: Chemax, 73: Tahdziú, 88: Teya, 85: Temozón y 58: Peto.

Gráfica 2. Mapeo de distancias entre municipios por niveles de pobreza e indicadores educativos

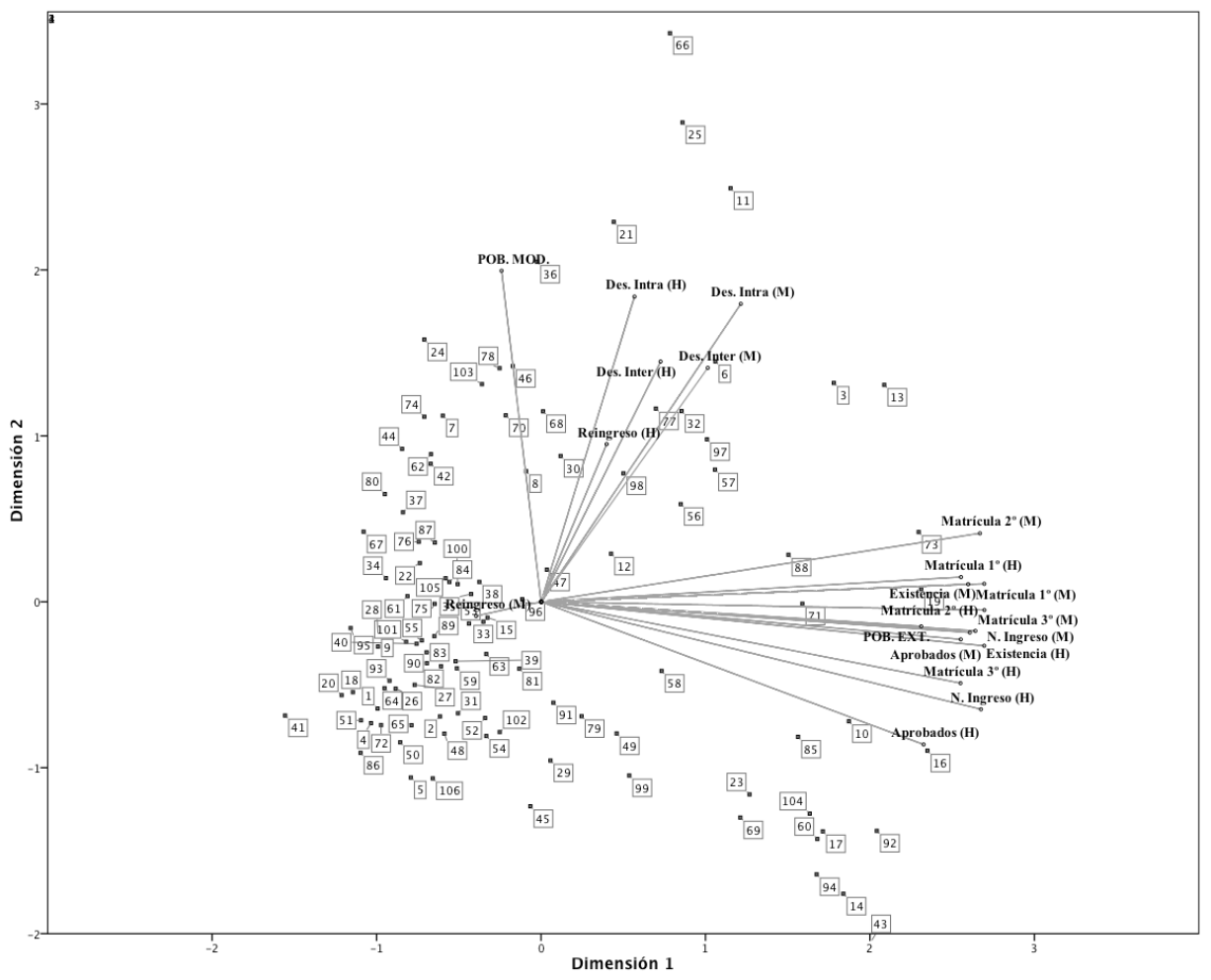




\section{DISCUSIÓN}

En relación con la eficiencia terminal se observa que, efectivamente, las zonas que presentan niveles de pobreza extrema tienen mayores niveles de eficiencia terminal. Aquellos municipios en pobreza extrema y que tuvieron índices más altos de eficiencia terminal fueron: Sudzal, Chacsinkin, Cantamayec, Chemax, Tahdziú, Teya, Temozón y Peto.

Esto se puede explicar, ya que la dificultad para permanecer en el sistema educativo en las comunidades con altos índices de pobreza se ha reducido debido a la existencia de apoyos por parte de programas gubernamentales como Oportunidades, que les permite continuar con sus estudios (Orazem y King, 2008). La Secretaría de Desarrollo Social, Sedesol (2010) reporta que a partir del año 2004 aumentó la probabilidad de entrar a la secundaria de los niños de zonas rurales de entre 12 y 14 años, $42 \%$ en el caso de los varones y $33 \%$ en el de las mujeres; así mismo reporta que la inscripción a secundaria se ha elevado 23\% a partir de 2002 en escuelas que cuentan con becarios de Oportunidades.

Por otra parte, aquellos estudiantes en condiciones de pobreza moderada son los que presentan mayores índices de deserción escolar. Las comunidades que presentan mayores índices de deserción escolar y cercanas al vector de pobreza moderada son: Dzan, Santa Elena, Dzitás, Celestún, Chichimilá y Espita.

Los datos sugieren que los alumnos dejan la escuela para dedicarse a otras actividades productivas de bienes y servicios en el ámbito local, ya que los apoyos económicos para continuar estudiando están dirigidos a zonas de alta marginación (INEE, 2009). Edmonds (2008) refiere que la pobreza se asocia con las distintas actividades que se dan al interior de cada comunidad, en función de las maneras que utilizan para la subsistencia alimentaria y las fuentes de empleo.

En este sentido, para la subsistencia y mantenimiento de las familias, los niños dedican horas de su tiempo escolar en labores domésticas o extra domésticas, por lo que asisten a la escuela con menor frecuencia que sus pares que no trabajan (Levinson, Moe, Knaul, 2008; Amin, Quayes, 
Rives, 2006). Lo cual podría ser más significativo en aquellos que están en situaciones de pobreza moderada.

Un ejemplo de esto es la población joven de Celestún, que, según estudios realizados en la zona, dejan la escuela para realizar actividades económicas, principalmente en sectores pesqueros o del ecoturismo (Córdoba, 2012); se le cataloga como "Reserva de la Biosfera Ría Celestún" (Semarnat, 2003). Lo cual, como mencionan García de Fuentes y Xool (2012), en ocasiones las diversificaciones turísticas o económicas de la zona pueden traer consecuencias sociales negativas, tal como la deserción o abandono escolar, ya que los niños dejan la escuela para incorporarse a las actividades turísticas de la zona.

En relación con la reincorporación de las niñas al nivel secundaria, el cual se asocia principalmente con aquellas que no se encuentran en situación de pobreza, puede explicarse en el sentido de que a la mujer en situación de pobreza, se le asignan funciones domésticas en los hogares como apoyo a la economía y subsistencia de los mismos. Este papel o rol asignado es inherente a ellas, aun cuando las niñas se desempeñen en otra actividad extradoméstica remunerada (Lamas, 1996). Esto implica que a las niñas que dejan la escuela para trabajar se les complica el reingreso a la escuela debido a los tiempos y demandas que deben cubrir para apoyar en la economía del hogar.

En Yucatán, según estadísticas del Consejo Estatal de Población (Coespo, 2011), viven cerca de 500 mil niños y niñas, de los cuales se estima que alrededor de 50 mil ejercen algún tipo de trabajo, esto es, 10\% de los niños de la entidad son económicamente activos. De los menores de 14 años en esta situación, según la Encuesta de Ocupación y Empleo (INEGI, 2011), más de 11 mil, es decir, 22.4\% no cumplen con la edad mínima requerida marcada por la Ley Federal del Trabajo. En 2011 en el grupo de cinco a 17 años, 33.3\% trabajaba en la industria, 23.1\% en el sector agropecuario, como artesanos o ayudantes, y $13.6 \%$ como comerciantes o empleados en comercios. Durante ese periodo, en el grupo de cinco a 17 años, $44 \%$ no asistía a la escuela, $32.7 \%$ trabajaba más de 35 horas a la semana y $34.6 \%$ no tenía ingresos o sus pagos eran en especie 


\section{CONCLUSIONES}

El presente artículo cumple con su objetivo, al reconocer que existen diferencias en cuanto a los niveles de pobreza y la deserción escolar en el nivel de secundaria, en los diversos municipios del estado de Yucatán. En este sentido, existen elementos que facilitan o limitan la permanencia en el sistema educativo, de acuerdo con los niveles de pobreza. Es decir, aquellos habitantes en situación de pobreza extrema tienen una alta probabilidad de recibir subsidios sociales como Oportunidades. Está documentado que dicho subsidio mejora sus condiciones generales de vida, aunque no garantiza que al terminal el nivel educativo exista oferta laboral (Coneval, 2012b). Por otro lado, aquellos habitantes ubicados en pobreza moderada no reciben los apoyos determinados por las políticas públicas y recurren a las ofertas en el mercado laboral o a la permanencia en el hogar para realizar aportes a la economía del mismo. Es principalmente en este nivel educativo que se da la mayor tasa de deserción debido, principalmente, a la edad, la cual es más adecuada en términos de desarrollo físico y emocional para ingresar al mercado laboral o apoyar en la familia.

También se concluye que en la pobreza moderada existe una diferencia significativa entre hombres y mujeres en cuanto al reingreso y deserción escolar. Las niñas tienden a quedarse en el hogar debido a que se considera, aun en nuestro contexto, que tienen un papel significativo en el ámbito privado y las labores domésticas y, como apoyo a la economía familiar, los roles se distribuyen entre los miembros de la familia. A diferencia de los varones que tienden a reincorporarse a la actividad escolar, ya que su función se dirige más hacia el ámbito público (Lagarde, 2012).

Este estudio arrojó datos significativos acerca de la relación entre los niveles de pobreza y deserción escolar en el nivel secundaria, al permitir datos actualizados de lo que sucede en los municipios del estado de Yucatán. 


\section{REFERENCIAS}

Abril, E., R. Roman, M. Cubillas e I. Moreno. 2008. ¿Deserción o autoexclusión? Un análisis de las causas de abandono escolar en estudiantes de educación media de Sonora, México, Revista Electrónica de Investigación Educativa, 10(1), <http://redie.uadc.mx/vol10nol/ contenido-abril.html>.

Alvarado, S., C. Leyva y R. Bolio. 2007. Estructura del ingreso y estrategias de subsistencia de la familia rural en la zona centro del estado de Yucatán, Economic Analysis Working Papers (2002-2010). Atlantic Review of Economics (2011-2013), 6, 1-47, <http://www.unagaliciamoderna.com /eawp/coldata/uplo ... iliaruralyucatan.pdf $>$.

Amin, S., S. Quayes y J. Rives. 2006. "Market work and household work as deterrents to schooling in Bangladesh", World Development, 34, 1271-1286.

Barkin, D. 2001. El turismo social en México: una estrategia necesaria, Actas Latinoamenricanas de Varsovia, 23, 7-30.

Becerril, J., R. Ortiz y L. Albornoz. 2012. "Maquiladoras e ingreso de los hogares en Yucatán", Problemas del Desarrollo, 171 (43).

Cámara de Diputados del H. Congreso de la Unión. 2013. Ley General del Desarrollo Social, Secretaría General, Secretaría de Servicios Parlamentarios.

Consejo Estatal de Población, Coespo. 2011. Secretaría General de Gobierno, Estado de Yucatán.

Consejo Nacional de Evaluación de la Política de Desarrollo Social, Coneval. 2010., Medición de la pobreza. Pobreza a nivel municipio 2010, $<$ http://www.coneval.gob.mx $>$ . 2012a. Informe de pobreza y evaluación en el estado de Yucatán. 2012b. Informe de evaluación de la política de desarrollo social en México, 2012.

. 2012c. Informe de pobreza en México. El país, el estado y sus municipios, 2010.

. 2013. Comunicado de prensa núm.003, Dirección de información y comunicación social. 
$<$ http://www.coneval.gob.mx/Informes/Coordinacion/Pobreza_2012/COMUNICADO_PRENSA_003_MEDICION_2012.pdf.>

Córdoba, A. 2012. De turisteros, ecoturistas y otros turistas en la comunidad de Celestún, Yucatán, México, en G. Marín, A. García de Fuentes y G.M. Daltabuit (coords.) Turismo, globalización y sociedades locales en la península de Yucatán, México, Pasos revista de turismo y patrimonio cultural, colección Pasos edita, núm. 7, cap. 8, 197-220, www.pasosonline.org.

Duro, E., M. Volpi y D. Contreras. 2010. La enseñanza secundaria en la región: logros y desafíos, en E. Duro (comp.) Educación secundaria: derecho, inclusión y desarrollo, Argentina, UNICEF.

Edmonds, E. 2008. Child labor, en T. Paul Schultz y John A. Strauss (comps.) Handbook of Development Economics, vol. 4, cap. 57, Amsterdam, North Holland.

Ferguson, K. 2006. “Social capital predictors of children's school status in México", International Journal of Social Welfare, 15, 321-331.

García de Fuentes, A. y M. Xool. 2012. Turismo alternativo y desarrollo en la costa de Yucatán, en G. Marín, A. García de Fuentes y G.M. Daltabuit (coords.) Turismo, globalización y sociedades locales en la península de Yucatán, México, Pasos revista de turismo y patrimonio cultural, colección Pasos edita, núm. 17.

Handa, S., H. Pineda, Y. Esquivel, B López, N. Guardian y F. Regalia. 2009. "Non-formal basic education as a development priority: Evidence from Nicaragua", Economics of Education Review. 28, 512522.

Instituto Nacional para la Evaluación de la Educación, INEE. 2009. Panorama educativo de México. Indicadores del sistema educativo nacional. Educación básica, México

Instituto Nacional de Estadística y Geografía. 2011. Encuesta Nacional de Ocupación y Empleo (ENOE). Indicadores de ocupación y empleo. Recuperado de http://www3.inegi.org.mx

Instituto Nacional de Estadística y Geografía. 2014. México en cifras. Información nacional, por entidad federativa y municipios. Recuperado de http://www.inegi.org.mx 
Lagarde, M. 2012. El feminismo en mi vida. Hitos, claves y topías, Instituto de las Mujeres de la ciudad de México, México.

Lamas, M. 1996. El género: la construcción cultural de la diferencia sexual, PUEG/Miguel Ángel Porrúa.

Levison, D., K. Moe y F. Knaul. 2008. "Making time: An analysis of youth hours of work and study in urban México", Review of Development Economics, 12 (4): 751-763.

Linting, M., J. Meulman, F. Groenen y A. Van der Kooij. 2007. Nonlinear principal components analysis: Introduction and application, Psychological Methods, 12(3): 336-358.

Meulman, J., A. Van Der Kooij y W. Heisser. 2004. Principal components analysis with notlinear optimal scaling transformations for ordinal and nominal data, en D. Kaplan (comp.) The sage handbook of quantitative methodology, Sage publications.

Orazem, P. y E. King. 2008. Schooling in developing countries: the roles of supply, demand and government policy, en T. P. Schultz y John Strauss (comps.), Handbook of Development Economics, vol. 4, cap. 55, Amsterdam, North Holland.

Pick, S. y J. Sirkin. 2010. Breaking the Poverty Cycle. The Human Basis for Sustainable Development, Oxford, Oxford University Press.

Petrakis, P. y D. Stamatakis. 2002. "Growth and educational levels: a comparative analysis", Economics of Education Review. 21, 513-521.

Roman, M. 2009. Abandono y deserción escolar: duras evidencias de la incapacidad de retención de los sistemas y su porfiada inequidad, Revista Iberoamericana sobre Calidad, Eficacia y Cambio en Educación, 7 (4): 3-9. <http://www.rinace.net/reice/numeros/arts/vol7num4/editorial.pdf $>$

Self, S. y R. Grabowski. 2004. "Does education at all levels cause growth? India a case study", Economics of Education Review, 23: 47-55.

Secretaría de Desarrollo Social. Sedesol. 2010. Oportunidades. Un programa de resultados, Gobierno Federal.

. 2012. Información del programa Oportunidades para directores y docentes de educación básica: ciclo 2012-2013, Gobierno Federal. 
Secretaría de Medio Ambiente y Recursos Naturales, Semarnat. 2003. Plan de manejo Reserva de la Biosfera Ría Celestún, Comisión Nacional de Áreas Naturales Protegidas, México, <http://www. semarnat.gob. $\mathrm{mx} / \mathrm{s}$.

Secretaría de Educación Pública, SEP. 2003. Diagnóstico general de la educación secundaria en el estado de Yucatán. 2005. Lineamientos para la formulación de indicadores educativos. Unidad de planeación y evaluación de políticas educativas, Dirección General de Planeación y Programación, México.

. 2006. Sistema de indicadores educativos de los Estados Unidos Mexicanos. Conjunto básico para el ciclo escolar 2004-2005. . 2012. Sistema educativo de los Estados Unidos Mexicanos. Principales cifras ciclo escolar 2011-2012. 